\title{
Assessment of interstitial mould growth through impaction: a feasibility study
}

\author{
Spyridon Efthymiopoulos ${ }^{1 *}$,Hector Altamirano ${ }^{1}$, and Valentina Marincioni ${ }^{1}$ \\ ${ }^{1}$ Institute for Environmental Design and Engineering, The Bartlett School of Environment, Energy and Resources, University College \\ London, UK.
}

\begin{abstract}
The application of insulation to solid walls is one of the measures to help reduce the energy consumption of a building. Where external wall insulation is not feasible (e.g. listed buildings), internal insulation may be the only option to improve the thermal property of external walls. However, such interventions may be followed by unintended consequences [1], such as moisture accumulation and the growth of mould [2]. The study aims to develop a method for the non-disruptive assessment of interstitial mould growth in internally insulated walls. Air sampling through impaction and culture-based analysis was used in the study. Mould species commonly found in buildings were cultivated and used in small-scale experiments, and the effects of several sampling periods and airflow rates assessed. Potential relationships between the sampling variables and the results were determined by means of statistical analysis - a strong correlation between the coverage of mould behind the wall and the sampling periods and airflow rates. The effect of the inertial impaction frequency on the sampling results and the feasibility and applicability of the suggested methodology in real-life scenarios are also discussed.
\end{abstract}

\section{Introduction}

In the UK, the built environment accounts for more than one-third of the total primary energy consumption and $\mathrm{CO}_{2}$ emissions [3]. Since more than $30 \%$ of the total heat losses of a building may occur through external walls [4], the installation of wall insulation (e.g. external, internal and cavity wall insulation) can considerably improve the thermal properties of the building envelope hence its energy performance. Yet, the installation of internal wall insulation (IWI) may be the only feasible solution in cases where, for example, the alteration of the façades of historic buildings is not permitted. However, moisture accumulation and condensation are thought to be two major risks that IWI interventions entail [5]. The phenomena may lead to serious unintended consequences such as interstitial mould growth, which may affect the structural integrity of the building and jeopardise the health of the occupants.

The present paper aims to examine the feasibility of mould growth assessment through the implementation of a novel air sampling and a culture-based technique.

\section{Literature}

The installation of IWI may be an efficient solution to reduce the heat losses of uninsulated wall, yet is not always a risk-free intervention. Interstitial moisture may appear due to the installation of materials with low vapour permeability [6], the improper installation of equipment, such as HVAC components, [7, 8], and when failing to seal around penetrations and services [8]. Furthermore, the improper installation of insulation at joist ends may contribute to moisture accumulation [9]. Mould growth and condensation due to moisture accumulation can cause structural damage, such as the decay of wood-based materials [10], leading to high restoration costs [11]. Health issues may also arise from the growth of interstitial mould. The World Health Organization (WHO) [12] suggests that mould contamination and dampness may affect occupant's wellbeing. Pulmonary infections may be caused by mould fragments, spores and particles that enter the human body through respiration [13, 14]. A causal relationship between mouldy environments and asthma affecting children has also been reported [11, 15]. Furthermore, Bush et al. [16] implied that health issues such as allergic bronchopulmonary aspergillosis (ABPA), allergic fungal rhinosinusitis (AFRS) and hypersensitivity pneumonitis (though uncommon) might be related to exposure to mouldy environments.

\subsection{Mould growth assessment}

Currently, several sampling procedures and analysis methods for the assessment of mould contamination are described in international guides and standards including Parts 16-20 of the BS ISO 16000 standard [17-21], the EMSL's Microbiology Sampling Guide [22], and the ASTM D7338-14 standard [23]. However, a specific assessment for "interstitial" mould has not been defined.

\footnotetext{
* Corresponding author: spyros.efthymiopoulos.18@ucl.ac.uk
} 


\subsubsection{Sampling methods}

Existing sampling methods can be divided into two main categories: (a) air sampling and (b) surface sampling. The most appropriate combination of sampling and analysis techniques should respond to the aim(s) of each investigation [24].

Air sampling methods are considered to be robust strategies for the assessment of mould's composition and the determination of its characteristics [25]. Often, air sampling procedures are divided into two groups: passive or non-aggressive sampling methods, which mainly capture airborne spores' fragments and microorganisms that pre-exist in the still air [26], and active or aggressive sampling methods, which relies on the resuspension of particles by disturbing the steadiness of the indoor air and surfaces [27].

Impaction, liquid impingement, spore trap sampling, cyclone sampling and air filtration are air sampling procedures that have been widely used. Among all other methods, air filtration is the only sampling technique that involves the collection of particles on a porous medium (filter) due to their size. All the other methods mentioned above include the collection of particles due to their inertia.

Selecting the most appropriate sampling procedure relies on the results that a particular study attempts to acquire. However, every method has its limitations, and thus the decision-making process may be challenging. Through sampling by impaction, particles are collected from the airstream created, on a sampling medium. Results can be extracted only when the collected spores, fragments or microorganisms are culturable. Through cyclone sampling and liquid impingement, both viable and non-viable particles can be collected, but bioefficiency losses can occur due to evaporation of the liquid and re-aerosolization of the particles [28].

Surface sampling methods involve the collection of samples to assess the growth of mould on surfaces. They are often used to examine whether the concentration of mould in the indoor environment is affected by fungal activity on surfaces [27]. Swab, wipe, bulk and tape lift are some commonly used surface sampling techniques. These methods are often selected to complement the air sampling procedures, and they contribute toward the assessment of contamination levels in the background.

The selection of an appropriate analysis technique is of key importance for the determination of the sampled mould's characteristics and the extraction of accurate, robust and high-quality results. Parts 16 to 20 of the BS ISO 16000 guide [17-21] describe the processing of data using various analysis methods. However, it is important to mention that limitations accompany every technique. Therefore, using more than one technique can enhance and maximise the accuracy of the assessment procedure [27]. WHO [12] has divided the analysis techniques into two main categories: (a) Culture-based methods and (b) Non-culture-based methods. The culture-based analysis relies on the cultivation of the culturable particles that were recovered during the sampling procedure. On the other hand, through non-culture-based methods, sampling data originating from either non-viable or viable particles that were collected during the sampling can be processed.

\section{Methodology}

The present study aims to examine the feasibility of assessing mould growing within confined spaces such as those created between external walls and IWI, using air sampling through impaction and culture-based analysis. Small scale experiments were conducted to develop an understanding of the relationship between the sampling variables (air flow rate and sampling period) and mould growth coverage. The data collected were processed through statistical analysis to identify potential links between the parameters of the sampling and the presence of mould.

\subsection{Experimental procedure}

The Aspergillus versicolor species was cultivated on six potato dextrose agar (PDA) plates for the purposes of the experiments. Optimum conditions were provided for the growth of the specific mould type (temperature and water activity of $27^{\circ} \mathrm{C}$ and 0.98 , respectively [29]) for 15 days before the experiments were conducted. The growth level of the mould was considered to be satisfactory for the experiments though the agar plates were not fully covered. It is essential to mention that according to the BS ISO 16000-17:2008 [18], the specific species can be used as an indicator of high humidity levels. As a result, in real case scenarios, enumeration of a high number of $A$. versicolor colonies after sampling, could signal the presence of high amount of interstitial moisture or the existence of potential leakage.

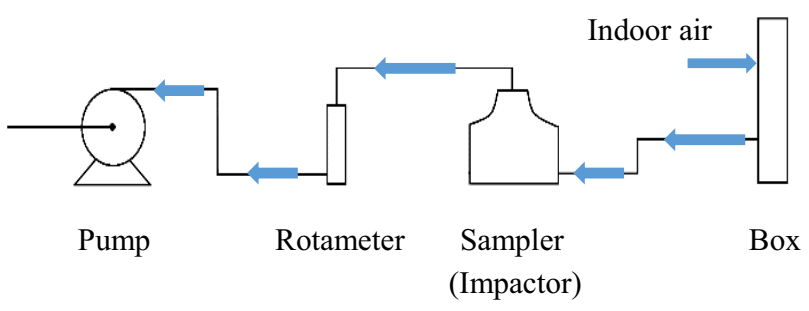

Fig. 1. Schematic representation of the experimental setup constructed, for the assessment of interstitial mould growth

A schematic representation of the setup constructed for the purposes of this research is shown in Fig. 1. A $45 \times 45 \times 5 \mathrm{~cm}$ box (Fig. 2) was used to simulate a confined space, which could be common in internally insulated walls in the UK. The dimensions were specifically selected so that 25 Petri dishes (Ø 90) could fit within the box. Two $6.35 \mathrm{~mm}$ holes were perforated $15 \mathrm{~cm}$ apart on the bisector of the acrylic surface of the box. The holes were perforated to allow the air to be driven from the indoor environment to the sampler. A pump was used to create an airstream within the box and force mould particles to be deposited on the medium of an impactor. A rotameter was used to measure the sampling flow rate and control the backpressure of the pump according to the needs of the experiments. It is important to mention that in real scale scenarios, creating two holes at the internal 
side of the wall under examination could ensure the avoidance of sampling air from the outdoor environment. In case that only one hole was perforated, due to the creation of a pressure differential by the pump, outdoor air could penetrate the solid wall through potential cracks, leading to the collection of air samples that could be misguiding.

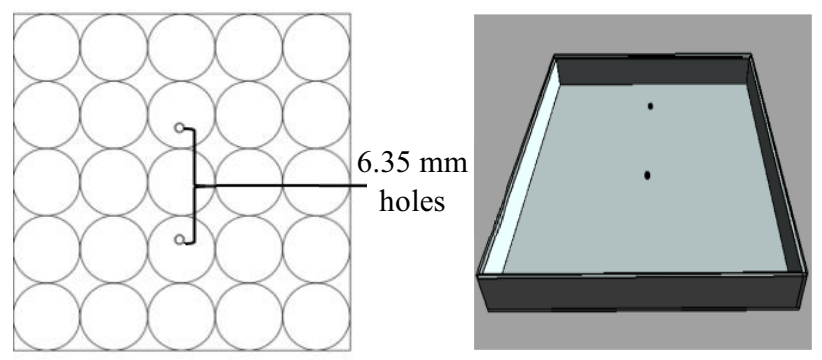

Fig. 2. Schematic representation of the box constructed for the experimental procedure and the location of perforated holes.

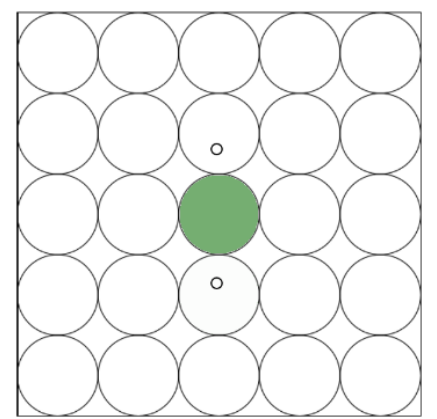

Fig. 3. Schematic representation of the mould plates' location within the experimental box during the experiments

All experiments were conducted inside an environmental chamber to reduce variations in the sampling results due to temperature and relative humidity fluctuations. The temperature and relative humidity within the chamber were set to be $25^{\circ} \mathrm{C}$ and $60 \%$ respectively and remained constant at all times. The specific values were selected so that uncertainties associated with particle desiccation or potential increase of spore production could be avoided. Low relative humidity may increase desiccation and thus reduce culturability while high relative humidity may increase the spore production rate. Thompson et al. [30] implied that the decrease of relative humidity from $90 \%$ to $30 \%$ during their experiments might have led to the reduction of culturable particles recovery rate.

The experiments were repeated four times to examine whether a statistically significant correlation between the airflow rate, the sampling time and the presence of mould could be identified. One plate with mould was placed on the middle of the box in every series as indicated in Fig. 3. Three sampling times ( $1 \mathrm{~min}, 2 \mathrm{~min}$ and $3 \mathrm{~min}$ ) and three airflow rates $(10 \mathrm{l} / \mathrm{min}, 20 \mathrm{l} / \mathrm{min}$ and $28.3 \mathrm{l} / \mathrm{min})$ were tested. These specific values were selected to complement previous analysis where it was found that small scale experiments should be repeated in order to extract results for statistical and mathematical purposes.

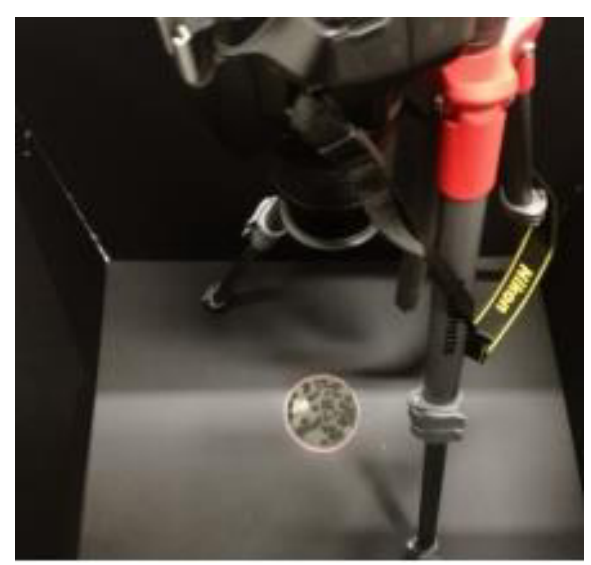

Fig. 4. Configuration created for the determination of the coverage of mould on each agar plate

The coverage of mould on each of the plates used for the four series of experiments conducted, was determined through image processing and simple programming scripts. Pictures of the four plates were taken using a DSLR camera and then processed through an image manipulation software. All pictures of the agar plates were taken under the same low lighting conditions to avoid reflections from the Petri dishes' surfaces. The configuration created to ensure the low lighting conditions is shown in Fig. 4.

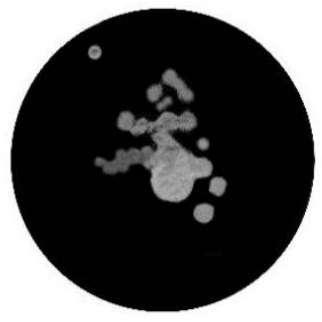

(a)

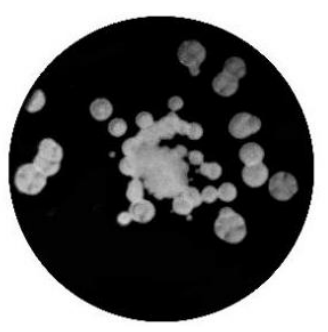

(c)

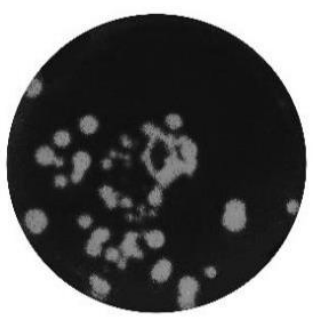

(b)

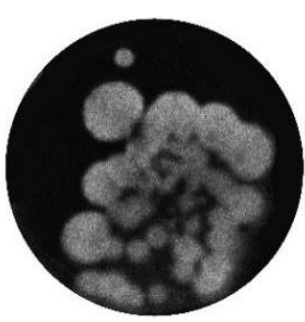

(d)
Fig. 5. Grayscale pictures of mould plates used all sets of experiments- (a) Series 1; (b) Series 2; (c) Series 3; (d) Series 4

Through image processing, the limits of the Petri dishes were identified, and the pictures were converted to grayscale (Fig. 5) so that they could easily be processed by the script created. The function created, relied on the conversion of the grayscale images into matrices, and the determination of the mould coverage was based on the colour differences. The procedure followed to determine the coverage of mould on every agar plate is illustrated in Fig. 6. 


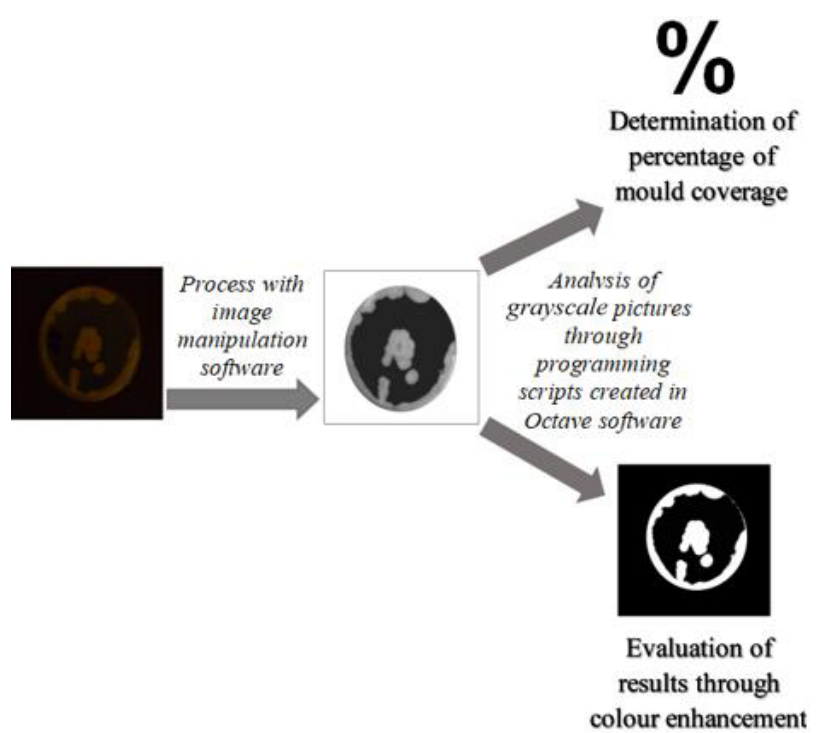

Fig. 6. Procedure to determine the percentage of mould coverage on the plates used for the four series of experiments surface covered by mould

During the 14 days cultivation period of the mould, a number of colonies merged with each other, making the enumeration of colony forming units (CFU) very difficult. To identify any potential relationships between the coverage of mould on each agar plate and the sampling results, the percentage of mould coverage variable was introduced. The specific variable refers to the percentage of the $45 \times 45 \mathrm{~cm}$ area (the surface of the box where the mould plate was placed) covered by mould.

The percentage of mould coverage within the box was calculated as the percentage of the plate surface that was covered by mould $\left(c_{i}\right)$ multiplied by the total area of the Petri dish $\left(0.00636 \mathrm{~m}^{2}\right)$ and divided by the total area of the box's surface $\left(0.2025 \mathrm{~m}^{2}\right)$ (Equation (1)).

$$
\text { Coverage }=\frac{c_{i} \cdot 0.00636}{0.2025}
$$

The mould coverage percentage for the 4 series of experiments that were conducted have been summarized in table 1.

Table 1. Percentage of mould coverage in every series of experiments

\begin{tabular}{|c|c|}
\hline $\begin{array}{c}\text { Series of } \\
\text { experiment }\end{array}$ & $\begin{array}{c}\text { Percentage of } \\
\text { mould coverage } \\
{[\%]}\end{array}$ \\
\hline 1 & 0.314 \\
\hline 2 & 0.628 \\
\hline 3 & 0.691 \\
\hline 4 & 1.476 \\
\hline
\end{tabular}

\section{Results analysis}

Four series of experiments were conducted to examine the effect of the sampling time. Fig. 7 summarizes the colonyforming units (CFU) per cubic metres of the sampled air. In all four series, the concentration of mould in the sampled air when the sampling time was set to 1 min was lower than the corresponding ones when the period was 2 $\min$. On the other hand, no clear pattern was identified in the results from the $3 \mathrm{~min}$ sampling procedures. However, it is important to mention that the findings from previous analysis showed that the $\mathrm{CFU} / \mathrm{m}^{3}$ values for a $3 \mathrm{~min}$ sampling period were lower than the $2 \mathrm{~min}$ period.

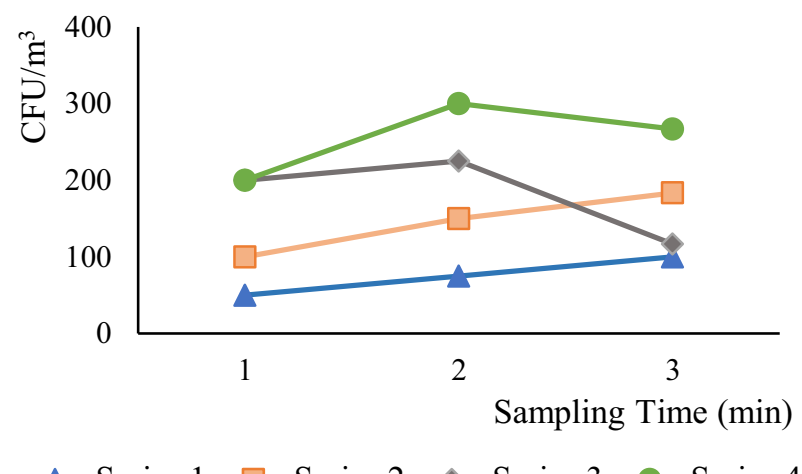

Fig. 7. Results of $\mathrm{CFU}$ concentration $\left(\mathrm{CFU} / \mathrm{m}^{3}\right)$ for the four series, for three different sampling times

The $\mathrm{CFU} / \mathrm{m}^{3}$ values measured from the experiments to determine the effect of the sampling air flow rate are illustrated in Fig. 8. It can be inferred that setting the flow rate to $10 \mathrm{l} / \mathrm{min}$ may not lead to an accurate assessment of the mould coverage.

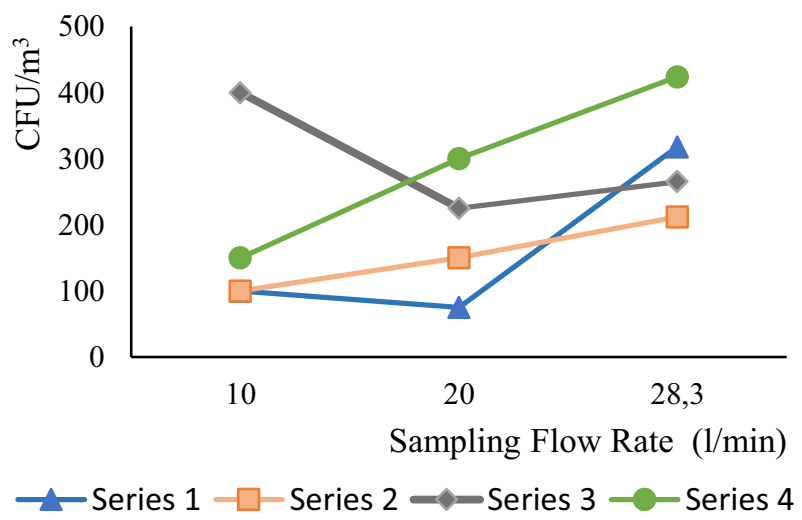

Fig. 8. Results of $\mathrm{CFU}$ concentration $\left(\mathrm{CFU} / \mathrm{m}^{3}\right)$ for the four series for three different air flow rates

The results extracted from the experimental sampling procedure were processed using statistical analysis. The Pearson correlation coefficient (r) was utilised to examine whether a potential correlation between the percentage of mould coverage from every series and the $\mathrm{CFU} / \mathrm{m}^{3}$ values for every sampling period and sampling velocity could be identified. The extracted coefficients are summarised in Table 2. Strong correlations between the sampling results and the data regarding the percentages of mould coverage were found for all the cases of different sampling times. No correlation was found between the coverage data and 
the data extracted when the airflow rate was set to 10 $1 / \mathrm{min}$. On the other hand, the $\mathrm{CFU} / \mathrm{m}^{3}$ values from the experiments where the flow rate was $28.3 \mathrm{l} / \mathrm{min}$ were found to have a strong correlation (0.704) to the percentage of mould coverage. Among all sets of data examined, the highest correlation $(0.927)$ between the size of the mould (expressed as percentage of mould covering the plates in every series of experiments) and the CFU $/ \mathrm{m}^{3}$ values measured was observed for the case where the sampling time and airflow rate were $2 \mathrm{~min}$ and $20 \mathrm{l} / \mathrm{min}$, respectively. However, the extracted results are also highly correlated to the coverage data, when the sampling time was set to $3 \mathrm{~min}$.

Table 2. Summary of the Pearson Correlation Coefficient determined for the sets of data examined during the experimental procedure

\begin{tabular}{|c|c|}
\hline Sets of Data & $\begin{array}{c}\text { Pearson } \\
\text { Correlation } \\
\text { Coefficient }(\boldsymbol{r})\end{array}$ \\
\hline $\begin{array}{c}\text { Percentage of mould coverage/Values } \\
\text { of CFU } / \mathrm{m}^{3} \text { for } 1 \mathrm{~min} \text { and } 201 / \mathrm{min}\end{array}$ & 0.759 \\
\hline $\begin{array}{c}\text { Percentage of mould coverage/Values } \\
\text { of CFU } / \mathrm{m}^{3} \text { for } 2 \mathrm{~min} \text { and } 201 / \mathrm{min}\end{array}$ & 0.927 \\
\hline $\begin{array}{c}\text { Percentage of mould coverage/Values } \\
\text { of CFU } / \mathrm{m}^{3} \text { for } 3 \mathrm{~min} \text { and } 201 / \mathrm{min}\end{array}$ & 0.913 \\
\hline $\begin{array}{c}\text { Percentage of mould coverage/Values } \\
\text { of CFU } / \mathrm{m}^{3} \text { for } 2 \mathrm{~min} \text { and } 101 / \text { min }\end{array}$ & 0.042 \\
\hline $\begin{array}{c}\text { Percentage of mould coverage/Values } \\
\text { of CFU } / \mathrm{m}^{3} \text { for } 2 \mathrm{~min} \text { and } 28.31 / \text { min }\end{array}$ & 0.704 \\
\hline
\end{tabular}

\subsection{Discussion}

An estimation of the coverage of the mould behind the wall may be possible if a flow rate of $20 \mathrm{l} / \mathrm{min}$ and a sampling time of 2 or 3 min are selected for the sampling procedure. On the other hand, it can be inferred that a lower airflow rate such as $10 \mathrm{l} / \mathrm{min}$ may not be appropriate for the assessment of interstitial mould.

Achieving a higher air velocity may have led to a more frequent occurrence of the inertial impaction phenomenon; the box constitutes a confined space; hence an increased number of mould particles may have been attached to the walls due to their inertia and the rapid changes in the airstream created. The increased frequency of the inertial impaction may lead to the assumption that the sampling results have been underestimated for the case where the flow rate was set to $28.3 \mathrm{l} / \mathrm{min}$. At the same time, as the turbulence within the setup increased, the frequency of the inertial impaction occurrence may have become more uncertain. As a result, the sampling results may have been affected, and the Pearson correlation coefficient may have been reduced in comparison to the corresponding value when the airflow rate was set to 20 $1 / \mathrm{min}$.

The results extracted from the samplings when the time period was $1 \mathrm{~min}$ were found to have a strong correlation $(0.759)$ to the set of data regarding the mould coverage percentage. However, selecting a sampling time of $1 \mathrm{~min}$ may not be appropriate for the assessment of interstitial mould, since longer sampling periods could better contribute to the collection of a more representative sample of the airborne fungus present within the box. As a result, a better estimation of the mould concentration in the sampled air may have been achieved, which consequently may have led to higher values of the correlation coefficient.

For the purposes of the research, the Aspergillus versicolor species was cultivated and used in all four sets of experiments. However, other species were also identified on the agar plates from the sampling. Full identification of the species recovered was not performed. The distribution of mould species and the number of CFU on the sampling medium is affected by the spore dispersal and the reproduction characteristics of each mould species [31]. Therefore, the suggestion of using a specific airflow rate and sampling times in this paper may be more appropriate for estimating the concentration of $A$. versicolor species in the sampled air than the moulds of other genera.

It is important to mention that the manufacturer of the impactor used for this research suggests that the median cut-point (D50) of the sampler is $0.6 \mu \mathrm{m}$. More than $60 \%$ of the airborne fungi in public buildings, offices, living spaces, and livestock farming have an aerodynamic diameter between $1 \mu \mathrm{m}$ and $5 \mu \mathrm{m}$ while the remaining percentage was found to have a diameter greater than $5 \mu \mathrm{m}$ [32]. According to a study by Marchisio et al. [33] the sizes of the Aspergillus versicolor particles lie between $2.1 \mu \mathrm{m}$ and $4.8 \mu \mathrm{m}$. Therefore, the Aspergillus versicolor species and a large percentage of fungi species can be collected by the impactor used for the experimental procedure.

Achieving high air velocities may reduce the bioefficiency of the sampler due to exposure of sensitive microorganisms to shear stress and thus reduction of their culturability [34]. Furthermore, Haig et al. [28] implied that high air flow rates may increase the frequency of the particle bounce phenomenon, which may result in the underestimation of the sampling results. The deposition build-up should also be of great concern as it may lead to loss of the sampler's bio-efficiency [28].

The period should be long enough to collect a representative sample of the airborne fungus [35]. However, selecting long sampling periods may lead to excessive desiccation and reduce the recovery capability of the sampling device [28,30,35-36].

All experiments concerning each agar plate with mould were conducted at 5-10 minutes intervals. During the specific period, the mould growth rate was considered very low and was assumed not to have a significant effect on the sampling results. However, successively conducting experiments may lead to a natural reduction of culturable particles. Preliminary results showed that experimenting with the same conditions five times may lead to small variations in the recovery rate of culturable particles. The results from the 2 nd and 4 th test were found to deviate the most from the result of the 1 st iteration, and the difference was less than $8 \%$. It was therefore assumed that the results extracted for the purposes of this study 
were not affected by the natural reduction of particles. Nevertheless, the results obtained are qualitative and further research to verify the assumption made should be conducted.

\section{Conclusions}

The air flow rate and sampling time were found to affect the sampling results. The relationship between the sampling parameters and the estimation of the concentration of mould in the sampled air may be connected to the frequency of inertial impaction. The increase of the airflow rate may have led to an increase of turbulence within the confined space and thus, more frequent occurrence of the inertial impaction phenomenon. The uncertainty accommodating the inertial impaction frequency may also have been increased by the additional turbulence; hence, selecting high air flow rates may lead to less accurate estimation of the coverage of the interstitial mould.

The selection of an appropriate sampling period was considered to be of great concern for the estimation of the concentration of mould in confined spaces, through impaction. Short sampling times may not be appropriate for the collection of sufficient data for the estimation of the interstitial mould coverage. On the other hand, sampling for a prolonged period of time may lead to desiccation stress and thus a reduction of the culturability and recovery of fungi particle on the sampling medium.

Selection of a sampling flow rate and period of 201/min and 2-3 min respectively was found to lead to high Pearson correlation coefficients between the coverage of the mould within the experimental box and the sampling results. Consequently, the detection of interstitial mould may be feasible in real case scenarios. However, the recovery of more than one species of mould during the experimental procedure suggests that air samples from the indoor environment should also be collected to assess the interstitial mould's coverage and characteristics more accurately.

Through the repeated experimental procedure and the determination of the Pearson correlation coefficient, a basic understanding of the relationship between the sampling time, air flow rate and the sampling results was developed. However, repetition of the experiments could contribute towards the collection of large samples of data that could be processed through statistical analysis and lead to more accurate and robust conclusions.

The present study aimed to examine the feasibility of interstitial mould assessment through air sampling by impaction and culture-based analysis. To this extent, the effect of sampling time and the flow rate to the sampling results were examined. However, the scope of this research is not limited to the identification of the most appropriate sampling flow rate and period. The analysis of the airflow pattern and the study of the effect of the location of mould and its composition on the sampling results is also crucial. Efthymiopoulos [37] has attempted to examine these aspects through computational fluid dynamics (CFD) analysis and small-scale experiments. The results of the research indicated that the perforation of the holes in a similar way to the one presented in section 3.1 could lead to the collection of particles from a large area within the confined space. Furthermore, the location of the mould was found to affect the results of the sampling, but the repetition of the testing procedure using both holes as inlet and outlet was suggested as a countermeasure to the issue. Nevertheless, the results of the research by Efthymiopoulos [37] are considered to be qualitative and can only indicate potential relationships between the aspects of concern and the sampling results. Consequently, further experiments and CFD analysis are encouraged to be conducted.

Further research to develop a better understanding of all the aspects that may affect the sampling results is recommended. Through small scale experiments, the effect of the temperature and humidity of the environment within the confined space, on the sampling results could be studied. Moreover, utilisation of more than one species for the experiments may provide insightful information regarding the effect of the mould's characteristics to the distribution and number of colonies recovered on the sampling medium.

Finally, the performance of real-scale experiments could inform on the applicability and feasibility of the suggested methodology in real scale constructions. As a result, a well-defined method for the assessment of interstitial mould growth could be determined. Such a technique could be standardised and used by professionals without the requirement for specialised training.

\section{References}

[1] C. Shrubsole, A. Macmillan, M. Davies, N May, 100 Unintended consequences of policies to improve the energy efficiency of the UK housing stock. INDOOR AND BUILT ENVIRONMENT, 23 (3) 340 - 352, (2014)

[2] N. May, Breathability: The Key to Building Performance, (2005)

[3] DECC. The Carbon Plan: Delivering our low carbon future, Energy, (2011)

[4] W. Anderson, J. Robinson, Warmer Bath: A guide to improving the energy efficiency of traditional homes in the city of Bath, Bath Preservation Trust and the Centre for Sustainable Energy, (2011)

[5] E. Biseniece, R. Freimanis, R. Purvins, A. Gravelsins, A. Pumpurs, \& A. Blumberga, Study of Hygrothermal Processes in External Walls with Internal Insulation, Environmental and Climate Technologies, 22(1), pp. 22-41, (2018)

[6] Ü. Alev, T. Kalamees, Avoiding mould growth in an interiorly insulated log wall. Building and Environment, 105, 104-115, (2016) 
[7] U.S. Environmental Protection Agency. Moisture Control Guidance for Building Design, Construction and Maintenance, pp. 1-20, (2013)

[8] BRE on behalf of CEW, Post installation performance of cavity wall \& external wall insulation. Constructing Excellence Wales, (2016)

[9] Changeworks, Solid Wall Insulation in Scotland. Exploring barriers, solutions and new approaches, pp. 5-18, (2012)

[10]BRE, Solid wall heat losses and the potential for energy saving, 44(0), pp. 136, (2014)

[11] W.J. Fisk, Q. Lei-Gomez, M.J. Mendell, Metaanalyses of the associations of respiratory health effects with dampness and mold in homes. Indoor Air, 17, pp. 284-296, (2007)

[12] World Health Organisation. Dampness and Mould. WHO Guidelines for Indoor Air Quality. Journal of Biomedical Semantics, 2011, pp. 2, 7-41

[13]B.J. Green et al., Airborne fungal fragments and allergenicity. Medical Mycology, 44 (Suppl. 1), pp. S245-S255, (2016)

[14] T.L. Brasel et al. Detection of airborne Stachybotrys chartarum macrocyclic trichothecene mycotoxins in the indoor environment. Applied and Environmental Microbiology, 71, pp. 7376-7388, (2005)

[15]D. Caillaud, B. Leynaert, M. Keirsbulck, R. Nadif, Indoor mould exposure, asthma and rhinitis: Findings from systematic reviews and recent longitudinal studies, European Respiratory Review, 27, pp. 1-18, (2018)

[16] R.K. Bush, J.M. Portnoy, A. Saxon et al. The medical effects of mold exposure. J Allergy Clin Immunol, 117: pp. 326-333. (2006)

[17]BSI, BS ISO 16000-16: 2008 Indoor Air: Detection and enumeration of moulds - Sampling by filtration, (2008)

[18]BSI, BS ISO 16000-17: 2008 Indoor Air: Detection and enumeration of moulds - Culture-based method, (2008)

[19]BSI, BS ISO 16000-18: 2011 Indoor Air: Detection and enumeration of moulds -Sampling by impaction, (2011)

[20]BSI, BS ISO 16000-19: 2014 Indoor Air: Sampling strategy for moulds, (2014)

[21]BSI, BS ISO 16000-20: 2014 Indoor Air: Detection and enumeration of moulds - Determination of total spore count, (2014)
[22] EMSL, MICROBIOLOGY SAMPLING GUIDE. EMSL Analytical, 1-28, (2014)

[23] ASTM. Standard Guide for Assessment Of Fungal Growth in Buildings, D7338 - 14, i, pp 1-9, (2014)

[24] T. Verdier, M. Coutand, A. Bertron, \& C. Roques, A review of indoor microbial growth across building materials and sampling and analysis methods. Building and Environment, 80, pp. 136-149, (2014)

[25]D. Méheust, et al., Indoor fungal contamination: health risks and measurement methods in hospitals, homes and workplaces. Critical Reviews in Microbiology, 40 (3), pp. 248-260, (2014)

[26] A.M. Swaebly, M.C. Christensen, Molds in house dust, furniture stuffing, and in the air within homes. J. Allergy Clin. Immunol. 23 (4), 370-374, (1952)

[27] S.C. Yang, et al., Sampling and analysis of indoor microorganisms / edited by Chin S. Yang, Patricia A. Heinsohn., Wiley-Interscience, (2007)

[28] W.C. Haig, G.W. Mackay, T.J. Walker, \& C. Williams, Bioaerosol sampling: sampling mechanisms, bioefficiency and field studies. Journal of Hospital Infection, 93(3), pp. 242-255, (2016)

[29] S.L. Smith, S.T. Hill, Infuence of temperature and water activity on germination and growth of Aspergillus restrictus and A. versicolor. Trans $\mathrm{Br}$. Mycol. Soc. 79 (3), 558-560, (1982).

[30] W.M. Thompson, et al. Method and test system for evaluation of bioaerosol samplers, Journal of Aerosol Science, 25(8), pp. 1579-1593, (1994)

[31]K. Tucker, et al. Biomechanics of conidial dispersal in the toxic mold Stachybotrys chartarum, Fungal Genetics and Biology, 44(7), pp. 641-647, (2007)

[32] M. Clauß, Particle size distribution of airborne micro- organisms in the environment - a review. (65), 77-100, (2015)

[33]F.V. Marchisio, R. Caramiello, L. Mariuzza, Outdoor airborne fungi: sampling strategies. AERIOBIOLOGIA5, 5(2), pp. 145-153, (1989)

[34] A. Adhikari, et al., Effect of different air flow rates on the collection of atmospheric mold spores of different sizes by a slit impactor, WIT Transactions on Ecology and the Environment, 211, pp. 243-253, (2017)

[35] S.A. Grinshpun, Biological Aerosols. In I. Agranovski, (ed), Aerosols - Science and Technology, Pp 379-396, (2010)

[36] G. Mainelis, M. Tabayoyong, The Effect of Sampling Time on the Overall Performance of Portable 
Microbial Impactors. Aerosol Science and Technology, 44. pp 75-82, (2010)

[37]Efthymiopoulos S. Assessment of mould growth within elements of exterior constructions. MSc dissertation. UCL. (2019) 